\title{
On the measurement of anisotropic elastic constants of fiber-reinforced composite plate using ultrasonic bulk wave and laser generated Lamb wave
}

\author{
T.-T. Wu *, Y.-H. Liu \\ Institute of Applied Mechanics, National Taiwan University, Taipei, Taiwan, ROC
}

Received 10 March 1999

\begin{abstract}
In this paper, we developed a hybrid elastic wave method to determine the anisotropic constants of a thin fiber-reinforced composite plate. The method is based on the measurements of the pure mode bulk ultrasonic wave velocities as well as the Lamb wave velocities. The formulae of the pure mode plane wave propagation in an orthotropic medium are summarized, first. Then, the numerical results of Lamb wave dispersion in a thin fiber-reinforced plate are presented. In the experimental part, conventional ultrasonic velocity measurements were conducted to obtain the pure mode velocities of a unidirectional fiber-reinforced composite and therefore the three corresponding elastic constants. The remaining two elastic constants of the thin composite plate were then obtained by using the inverse analyses of the Lamb wave dispersion. We note that with this hybrid method, some of the anisotropic constants can be measured accurately with well-developed bulk wave ultrasonics, while the rest of the undetermined elastic constants can be obtained from the inversion of the Lamb wave dispersion. (C) 1999 Elsevier Science B.V. All rights reserved.
\end{abstract}

Keywords: CFRP; Lamb wave; Laser ultrasonics; Non-destructive evaluation

\section{Introduction}

Fiber-reinforced composite materials have been utilized extensively in industrial applications, such as ship and aircraft engineering. Recently, they have also been applied to the retrofit of concrete structures. The key feature of fiber-reinforced composite material is its high strength to weight ratio. The anisotropy of the material allows the strength to be utilized in a given design direction without the addition of extra weight resulting from strength in unnecessary directions. However, the complex constitutive nature of composite materials leads to difficulties in the determination of their mechanical properties [1]. By conventional mechanical testing techniques, the Young's moduli along different principal axes of symmetry of fiber-reinforced composites must be measured through different uniaxial tests. In addition to the Young's moduli, the measurements of the shear moduli of fiber-reinforced composites are also not easy, the rail shear or torsion tube test must be invoked.

Ultrasonic techniques have been applied successfully

\footnotetext{
* Corresponding author. Tel.: + 886-2-2363-0979; fax: + 886-2-2363-9290.

E-mail address: wutt@spring.iam.ntu.edu.tw (T.-T. Wu)
}

in the field of non-destructive evaluation of materials. Since fiber-reinforced composite materials are anisotropic in nature, the conventional ultrasonic technique for measuring material constants in isotropic materials cannot be utilized to obtain all of the anisotropic elastic constants. In the literature, there are extensive researches related to the study of ultrasonic waves in composite laminates. Datta et al. [2] utilized the stiffness method to study dispersive wave propagation in a laminated anisotropic plate. Rose et al. [3] utilized Lamb waves to evaluate the anisotropic properties of graphite-epoxy composites. Dayal and Kinra [4] examined the dispersion relations of the leaky Lamb waves in an anisotropic plate and developed an experimental technique for measuring phase velocity and attenuation of ultrasonic waves in thin plates. The propagation of free waves in plates of general anisotropic materials was studied by Nayfeh and Chimenti [5]. The experimental study of the propagation of horizontally polarized shear waves in a unidirectional thin fiber-reinforced composite plate was made by $\mathrm{Wu}$ and Chiu [6]. Laser generated ultrasonic surface waves [7] have also been applied to measure the elastic constants of anisotropic half space. The anisotropic elastic constants were obtained using the inverse analyses 
of the measured group velocities of laser generated surface waves. Veidt and Sachse [8] measured the stiffness tensor of thin fiber-reinforced laminates based on the point-source/point-receiver technique. A simple plane wave, plane stress model was utilized to describe the quasi-longitudinal and quasi-transverse waves. On the other hand, there are researches on the measurements of elastic properties of thin plates using Lamb waves [9-12], but most of the test specimens are isotropic.

In this paper, we developed a hybrid elastic wave method to determine the anisotropic constants of a thin fiber-reinforced composite plate. The method is based on the measurements of the pure mode bulk ultrasonic wave velocities as well as the Lamb wave velocities. In the following, the formulae of the pure mode plane wave propagation in an orthotropic medium are summarized, first. Then, the numerical results of Lamb wave dispersion in a thin fiber-reinforced plate are presented. In the experimental part, the conventional ultrasonic velocity measurements were conducted to obtain the pure mode velocities of a unidirectional fiber-reinforced composite and then the corresponding elastic constants. Finally, laser ultrasonic experiments were conducted to obtain the Lamb wave dispersion and an inverse algorithm was utilized to determine the remaining two elastic constants of the thin unidirectional composite plate.

\section{Bulk ultrasonic wave measurement}

\subsection{Plane waves in orthotropic media}

For an anisotropic linear elastic material with no body force, the equation of motion for the wave displacement $u_{i}$ has the form:

$C_{i j k l} \frac{\partial^{2} u_{i}}{\partial x_{j} \partial x_{l}}=\rho \frac{\partial^{2} u_{i}}{\partial t^{2}}$

where $C_{i j k l}$ are the elastic constants and $\rho$ is the mass density. A plane wave propagating in the medium can be represented as:

$u_{i}=U_{i} \exp \left[\mathrm{i} k\left(n_{j} x_{j}-V t\right)\right]$

where $U_{i}$ is the amplitude, $k$ the wave number, $n_{j}$ the wave normal, and $V$ the phase velocity of the plane wave. On substituting Eq. (2) into Eq. (1), the phase velocity and wave amplitude must satisfy the following system of equations:

$\left.C_{i j k l} n_{j} n_{l}-\rho V^{2} \delta_{i k}\right) U_{k}=0$

where $V$ is determined from the characteristic equation:

$\left|C_{i j k l} n_{j} n_{l}-\rho V^{2} \delta_{i k}\right|=0$.
Consider an orthotropic medium where the planes of orthotropic symmetry are taken as the coordinate planes; it is easy to find that for plane waves propagating in the direction of the axis of orthotropic symmetry, the motion may be either a pure longitudinal or a pure transverse wave. Therefore, six independent pure mode waves exist, three longitudinal and three shear. Let $V_{i j}$ denote a wave propagating along the $x_{i}$-axis with polarization in the direction of the $x_{j}$-axis, then the six wave speeds can be expressed in matrix form as [13]:

$\left[\rho V_{i j}^{2}\right]=\left[\begin{array}{lll}C_{11} & C_{66} & C_{55} \\ C_{66} & C_{22} & C_{44} \\ C_{55} & C_{44} & C_{33}\end{array}\right]$

where the elastic constants have been written in Voigt's contracted notation, in which 11 is replaced by 1,22 by 2,33 by 3,23 by 4,31 by 5 , and 12 by 6 .

\subsection{Pure mode waves in a unidirectional thin fiber- reinforced composite laminate}

A unidirectional fiber-reinforced composite thin plate can be assumed as in transversely isotropic symmetry, and hence, the independent elastic constants are reduced from nine (orthotropic) to five. Let the $x_{1}$-axis be pointed along the fiber direction, then the $x_{2}-x_{3}$ plane is the isotropic plane (Fig. 1). The non-vanishing elastic constants are:

$\left[C_{i j}\right]=\left[\begin{array}{cccccc}C_{11} & C_{12} & C_{12} & 0 & 0 & 0 \\ C_{12} & C_{33} & C_{23} & 0 & 0 & 0 \\ C_{12} & C_{23} & C_{33} & 0 & 0 & 0 \\ 0 & 0 & 0 & C_{44} & 0 & 0 \\ 0 & 0 & 0 & 0 & C_{55} & 0 \\ 0 & 0 & 0 & 0 & 0 & C_{55}\end{array}\right]$

where $C_{44}=\left(C_{33}-C_{23}\right) / 2$.

From Eq. (5), we understand that for wave propagation in such an anisotropic plate, pure wave mode

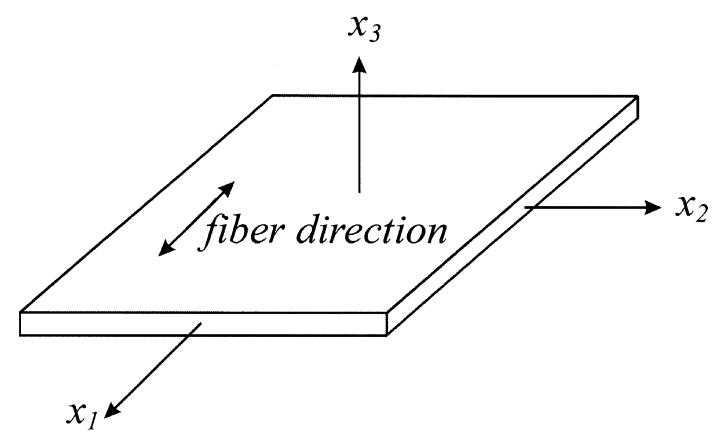

Fig. 1. The coordinates of the thin fiber-reinforced laminates. 
(longitudinal or transverse) exists only when the propagation direction is along or perpendicular to the fiber direction. For all the other propagation directions, the wave modes are quasi-longitudinal or quasi-transverse. We note that for bulk ultrasonic wave measurement in such a thin plate, the only accessible plane is the $x_{1}-x_{2}$ plane (see Fig. 1), and therefore, it is not feasible to measure the phase velocity with propagation direction along $x_{1}$ - or $x_{2}$-direction. For wave normal pointed along the $x_{3}$-direction, the relations between the phase velocities and the elastic constants can be obtained from Eq. (5) as:

$V_{31}=\sqrt{\frac{C_{55}}{\rho}}, V_{32}=\sqrt{\frac{C_{44}}{\rho}}, V_{33}=\sqrt{\frac{C_{33}}{\rho}}$.

In the above, $V_{31}$ and $V_{32}$ are transverse (shear) wave speeds with polarization directions along the $x_{1}$ - and $x_{2}$-directions, respectively. $V_{33}$ is the longitudinal wave speed.

From Eq. (7), we note that three out of five independent elastic constants of a transversely isotropic material can be determined from the conventional ultrasonic bulk wave measurements. We note that with the current available ultrasonic velocity measurement technique, the aforementioned three wave speeds can be measured accurately. The purpose of this paper is to utilize the Lamb wave propagation in a thin plate to determine inversely the remaining unknown elastic constants $C_{11}$ and $C_{12}$.

\section{Dispersion of Lamb wave in a thin transversely isotropic plate}

Different from an infinite space, a thin plate preserves two parallel flat boundaries, the elastic wave motions on each surface will interact to produce Lamb waves. The phase velocity of Lamb wave is varied according to the distance between the two boundaries, and therefore, the wave is dispersive. The basic modes of Lamb waves are the symmetric and antisymmetric modes. It is worth noting that for receiving distance sufficiently far away from the source, most of the Lamb wave energy is dominated by the first antisymmetric mode [14]. This means that the dispersion of the first antisymmetric Lamb mode can be obtained directly from the spectral analysis of the time domain Lamb wave signal in a thin plate with reasonable accuracy.

In this section, we calculate the phase velocity dispersion of Lamb wave propagation in a thin unidirectional fiber-reinforced composite. Two cases are considered, one is for a Lamb wave propagating along the fiber direction, the other is for a Lamb wave propagating normal to the fiber direction. A general-purpose computer program for the calculation of phase velocity

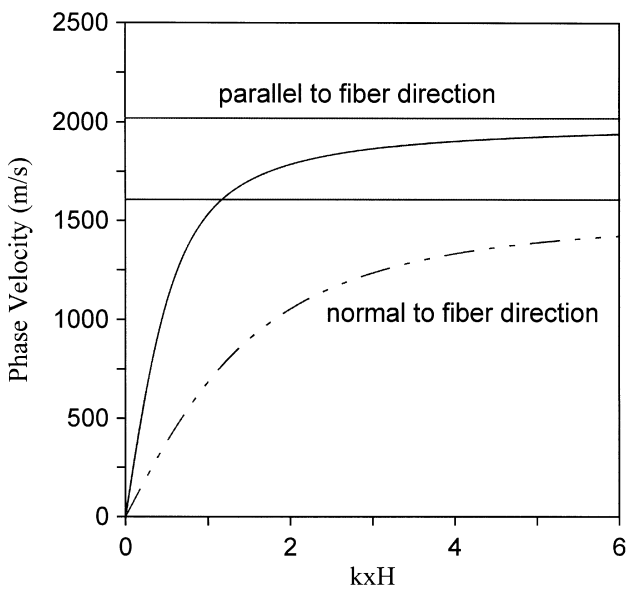

Fig. 2. The dispersion curves for the antisymmetric Lamb waves propagating parallel (solid line) and normal (dotted line) to the fiber direction.

dispersion in a multi-layered anisotropic medium was utilized. The program was based on the sextic formalism of Stroh $[15,16]$ for the calculations of dispersion curves of anisotropic multi-layered media.

Shown in Fig. 2 are the dispersion curves for the antisymmetric Lamb waves propagating parallel (solid line) and normal (dotted line) to the fiber direction. The material properties of the unidirectional carbon fiberreinforced composite utilized were adopted from Ref. [7]. The thickness of the plate is $H$. The results show that for both Lamb propagation directions (parallel or normal to the fiber directions), the high frequency component propagates faster than the low frequency component, which is similar to that of the isotropic case. In addition, the propagation speed for a Lamb wave propagating parallel to the fiber direction is faster than that normal to the fiber direction.

\section{Conventional bulk ultrasonic measurements}

The carbon fiber-reinforced composite specimen used is a 14-prepreg layered plate with fibers oriented along the $x_{1}$-direction. The thickness of the composite plate is $2.25 \mathrm{~mm}$, and the mass density is $1520.4 \mathrm{~kg} / \mathrm{m}^{3}$.

As described in the previous section, for a transversely isotropic material, three elastic constants $\left(C_{55}, C_{44}\right.$, $C_{33}$ ) can be measured easily using the conventional ultrasonic measuring technique. Therefore, we measured the phase velocities for waves normal along the $x_{3}$-direction, i.e. $V_{31}, V_{32}, V_{33}$. Then, from Eq. (7) and the known density of the specimen, $C_{55}, C_{44}, C_{33}$ are obtained.

In the measurements, longitudinal wave and transverse wave transducers with $5 \mathrm{MHz}$ center frequency were used. The diameter of the transducers is 
0.25 in. The measured results are:

$V_{31}=1810.9 \mathrm{~m} / \mathrm{s}, \quad V_{32}=1431.0 \mathrm{~m} / \mathrm{s}, \quad V_{33}=2735.6 \mathrm{~m} / \mathrm{s}$.

The corresponding elastic constants can thus be obtained as:

$C_{22}=C_{33}=11.38 \mathrm{GPa}, C_{55}=C_{66}=4.99 \mathrm{GPa}$

$C_{44}=3.11 \mathrm{GPa}, C_{23}=5.15 \mathrm{GPa}$.

\section{Laser ultrasonic measurements}

Shown in Fig. 3 is the laser ultrasonic experimental set-up utilized in this study. An Nd:YAG pulsed laser (Quanta-Ray, GCR-130) (wavelength $532 \mathrm{~mm}$ ) was utilized to generate an elastic wave in the layered specimen. The duration of the laser pulse utilized was $10 \mathrm{~ns}$ and the energy carried was about $100 \mathrm{~mJ}$. The composite specimen was rested on a precision translation stage to accurately control the distance between the source and the receiver. An NBS conical transducer was utilized to measure the generated elastic wave signals from the laser sources. The received voltage signals from the conical transducer were then amplified by a preamplifier and recorded by a digital oscilloscope. A trigger signal synchronized with the laser source was utilized to trigger the digital oscilloscope. The recorded signals were sent to a personal computer via GPIB.

\subsection{Lamb wave in a unidirectional composite thin plate}

An isotropic copper thin plate was used to test the experimental set-up first. The density of the $\mathrm{Cu}$ plate is $8500 \mathrm{~kg} / \mathrm{m}^{3}$ and the thickness is $1 \mathrm{~mm}$. The longitudinal and transverse wave speeds are 4545.5 and $2222.2 \mathrm{~m} / \mathrm{s}$, respectively. Shown in Fig. 4 are the waveforms for a Lamb wave in such a thin plate. The source-receiver distances are 4 and $6 \mathrm{~cm}$. The results showed that the symmetric mode arrives earlier (at approximately $5 \mu \mathrm{s}$ for the case of $4 \mathrm{~cm}$ source-receiver distance in Fig. 4) than the antisymmetric mode, while the amplitude of the symmetric mode is much smaller than that of the antisymmetric mode. Further, in the antisymmetric mode signals, the dispersive nature of the signal is very clear, i.e. higher frequency signal arrives earlier than lower frequency signal.

The same experimental set-up was then utilized to study the propagation of Lamb waves in the unidirectional fiber-reinforced composite thin plate that was mentioned in the previous section. Shown in Fig. 5 are the waveforms of Lamb waves propagating normal to the fiber direction. The source-receiver distance was varied from 3 to $7 \mathrm{~cm}$, i.e. from about 14 plate thicknesses to 30 plate thicknesses. For the case of sourcereceiver distance equal to $3 \mathrm{~cm}$, the measured waveform shows that the displacement signal is dominated by the lowest order antisymmetric mode. The dispersive nature of the signal is not very clear at this range. At the larger source-receiver distance, say $7 \mathrm{~cm}$, the waveform shows

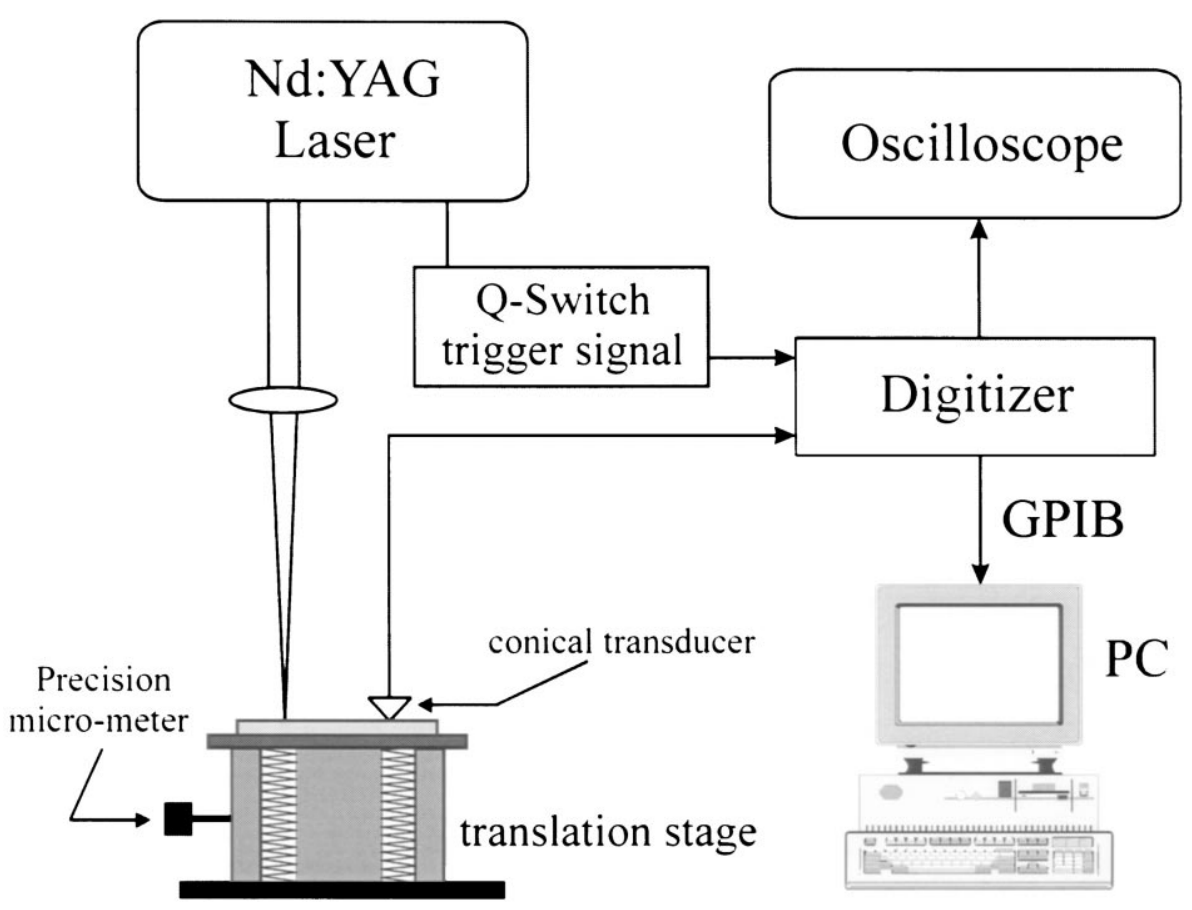

Fig. 3. Experimental set-up of the laser ultrasonic experiment. 


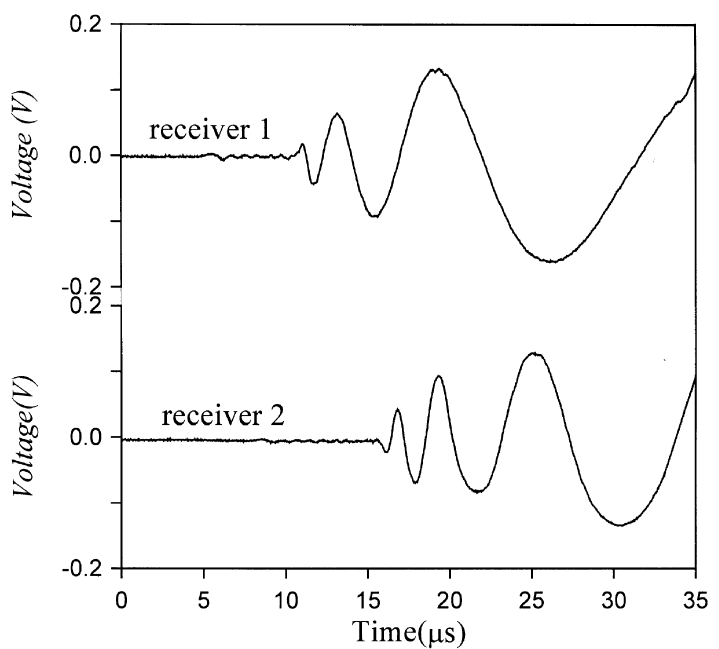

Fig. 4. The waveforms of Lamb wave in an anisotropic thin $\mathrm{Cu}$ plate.

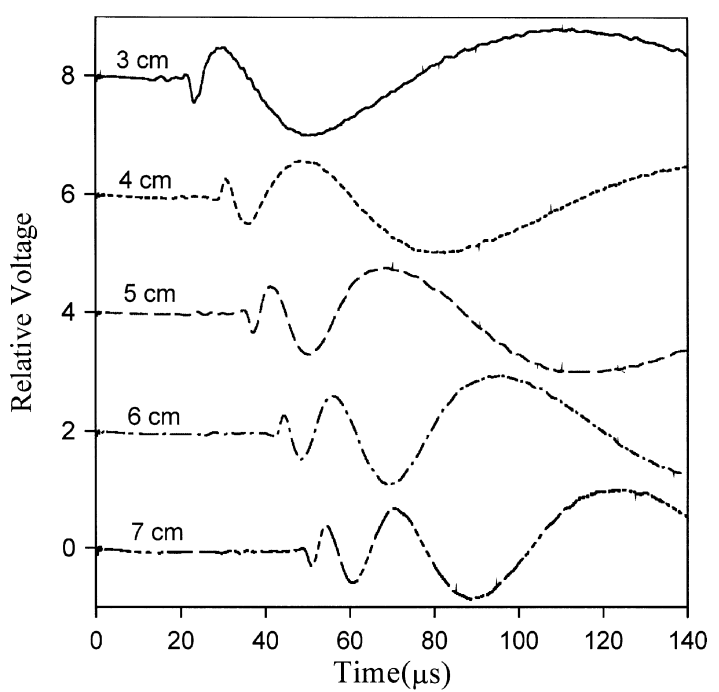

Fig. 5. The waveforms of Lamb waves in the thin fiber-reinforced composite plate with propagating direction normal to the fiber direction.

clear dispersive characteristics of the Lamb wave signal. From Fig. 5, one can find the evolution of the dispersive waveforms as a function of the source-receiver distance.

Fig. 6 shows the waveforms of Lamb waves propagating parallel to the fiber direction with the source-receiver distance varied from 3 to $7 \mathrm{~cm}$. These waveforms show that the normal displacement of the Lamb wave is dominated again by the first order antisymmetric mode, however, the dispersive nature of the waves are not so clear as those of Fig. 5, in which the Lamb wave is propagating normal to the fiber direction. In fact, the waveform for the case of $7 \mathrm{~cm}$ source-receiver distance in Fig. 6 is similar to that of $3 \mathrm{~cm}$ source-receiver distance in Fig. 5. Therefore, we note that the Lamb wave needs a larger source-receiver distance to show the dispersive nature when the propagating direction is parallel to the fiber direction.

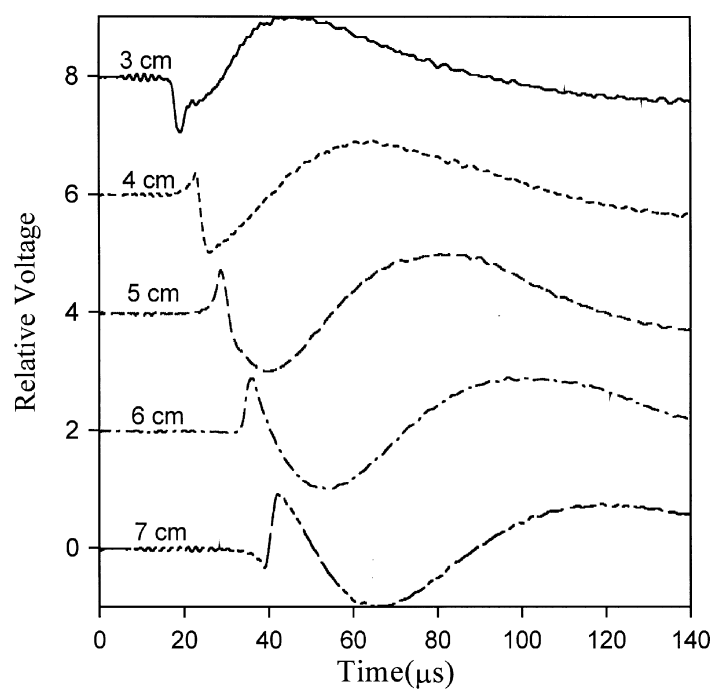

Fig. 6. The waveforms of Lamb waves in the thin fiber-reinforced composite plate with propagating direction parallel to the fiber direction.

To understand the characteristics of Lamb wave propagation in different directions, we fixed the sourcereceiver distance at $6 \mathrm{~cm}$ and varied the propagation angle from $0^{\circ}$ (parallel to the fiber direction) to $90^{\circ}$ (normal to the fiber direction). The experimental results shown in Fig. 7 show clearly the evolution of the dispersive nature of Lamb wave propagation direction from $0^{\circ}$ to $90^{\circ}$ in the thin fiber-reinforced composite plate.

\subsection{Spectral analysis of Lamb waves}

The phase difference $\phi$ of two wave signals received at two different positions $x_{1}$ and $x_{2}$ is equal to the phase

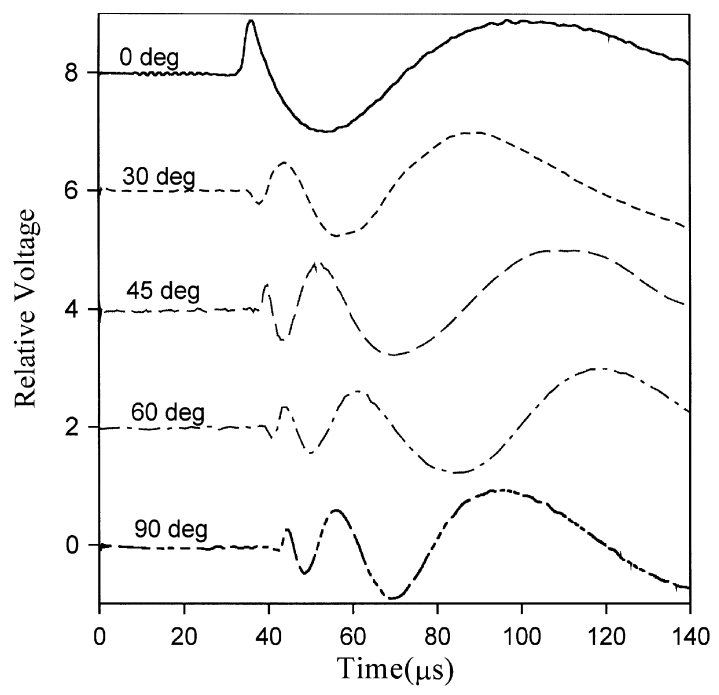

Fig. 7. The waveforms of Lamb waves with propagation direction varied from $0^{\circ}$ to $90^{\circ}$ in the thin fiber-reinforced composite plate. 


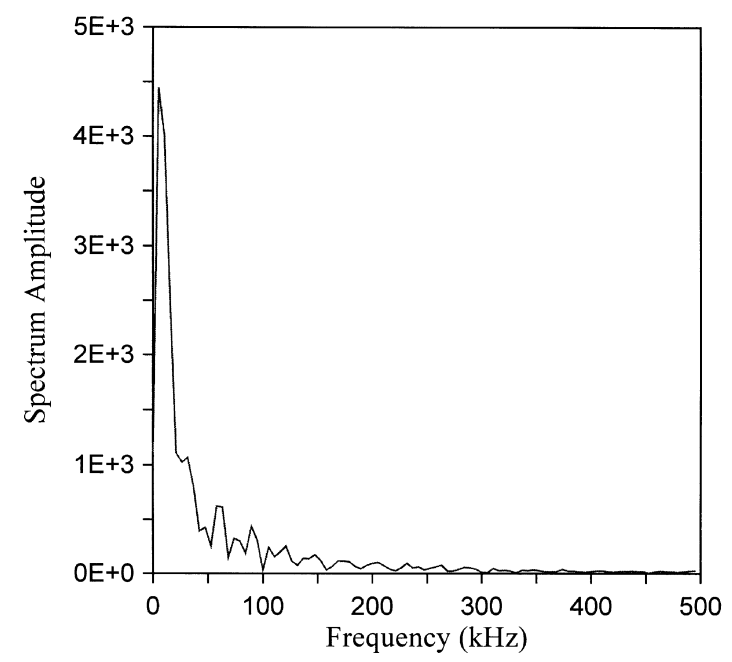

Fig. 8. The frequency spectrum of the wave signal in Fig. 6 for the case of source-receiver distance equal to $6 \mathrm{~cm}$.

angle of $S_{x_{1}, x_{2}}$, which is defined as:

$S_{x_{1}, x_{2}}(f)=R_{1}(f) R_{2}^{*}(f)$.

In the above equation, $R_{1}(f), R_{2}(f)$ are the frequency spectrum of the wave signals received at two different positions $x_{1}$ and $x_{1}, f$ is the frequency of the harmonic wave, $R_{2}^{*}$ denotes the complex conjugate of $R_{2}$. The phase velocity $v$ can then be obtained from the relation:

$v=2 \pi f \frac{x_{1}-x_{2}}{\phi}$.

Fig. 8 shows the frequency spectrum of the wave signal in Fig. 6 for the case of source-receiver distance equal to $6 \mathrm{~cm}$. The propagation direction of the Lamb wave is along the fiber direction. The result shows that the maximum frequency of the received signal is around $300 \mathrm{kHz}$. A similar result was obtained for the case of source-receiver equal to $4 \mathrm{~cm}$. Utilizing Eqs. (8) and (9), the dependence of the phase velocity of the Lamb wave on the frequency is shown by square symbols in Fig. 9. The solid circles in Fig. 9 are the dispersion of a Lamb wave propagating perpendicular to the fiber direction. We note that the experimental results reflect the dispersion relations of the theoretical results shown in Fig. 2.

\section{Inversion of anisotropic elastic constants}

For the coordinate system shown in Fig. 1, we have shown that $C_{33}, C_{44}$ and $C_{55}$ of a transversely isotropic material could be measured using conventional bulk ultrasonics. The remaining unknown elastic constants are $C_{11}$ and $C_{12}$. For waves propagating in the

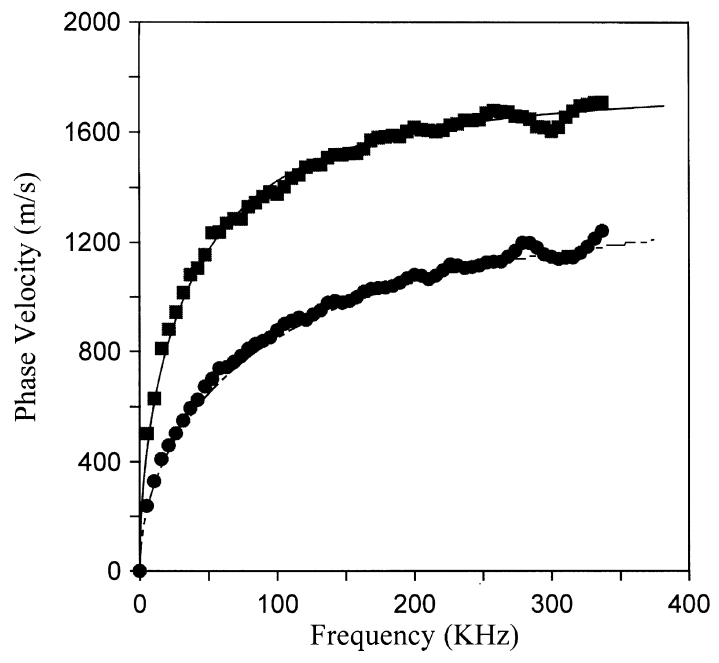

Fig. 9. Experimental dispersion of the antisymmetric Lamb waves propagating parallel (square symbols) and normal (solid circles) to the fiber direction.

$x_{2}$-direction, it is easy to show that $C_{11}$ and $C_{12}$ do not appear in the Christoffel equation and hence the dispersion relation either. Therefore, only the experimental dispersion relation for a Lamb wave propagating along the fiber direction shown in Fig. 9 can be utilized to determine $C_{11}$ and $C_{12}$.

\subsection{Inversion scheme}

An error function which defines the difference between the measured $\left(v_{\mathrm{m}}\right)$ and the guessed $\left(v_{\mathrm{g}}\right)$ phase velocities was defined as:

$e=\frac{\sum_{i=1}^{N}\left[v_{\mathrm{m}}(i)-v_{\mathrm{g}}(i)\right]^{2}}{\sum_{i=1}^{N}\left[v_{\mathrm{m}}(i)\right]^{2}}$

where $i$ represents the discrete non-dimensional wave number and $N$ is the number of data points utilized in the inversion process.

In the inversion process, initial guesses of $C_{11}$ and $C_{12}$ were made first, then the forward computer program for calculating the phase velocity dispersion of a Lamb wave in a transversely isotropic plate was utilized to calculate the guessed phase velocities $\left(v_{\mathrm{g}}\right)$. The value of the error function can thus be obtained from Eq. (10). The true elastic constants, $C_{11}$ and $C_{12}$, were then determined using the simplex method $[17,18]$.

\subsection{Inversion results}

The measured dispersion relation for Lamb waves propagating along the fiber direction shown in Fig. 9 
was utilized to determine inversely the elastic constants $C_{11}$ and $C_{12}$. Since there are two unknowns to be determined, three initial guesses have to be made. To test the influence of the initial guesses on the inversely determined results, four different sets of initial guesses were used and the results are shown in Table 1 . We note that although the initial guesses of $C_{11}$ and $C_{12}$ range from around $60 \%$ to $200 \%$ of the true values, the inverse results converge nicely. The average values of the inversely determined constants $C_{11}$ and $C_{12}$ are $C_{11}=134.45 \mathrm{GPa}, C_{12}=7.96 \mathrm{GPa}$. The standard deviation of $C_{11}$ is $0.17 \%$ of the average value, while that of $C_{12}$ is $2.4 \%$. Therefore, on combining with those values measured by the ultrasonic bulk wave method, the elastic constants of the unidirectional fiber-reinforced composite laminates are determined as:

$\left[C_{i j}\right]=\left[\begin{array}{cccccc}134.45 & 7.96 & 7.96 & 0 & 0 & 0 \\ 7.96 & 11.38 & 5.15 & 0 & 0 & 0 \\ 7.96 & 5.15 & 11.38 & 0 & 0 & 0 \\ 0 & 0 & 0 & 3.11 & 0 & 0 \\ 0 & 0 & 0 & 0 & 4.99 & 0 \\ 0 & 0 & 0 & 0 & 0 & 4.99\end{array}\right]$ GPa.

The solid lines in Fig. 9 are the dispersion relations calculated based on the elastic constants shown in Eq. (11). We note that the experimental results are in accordance with the theoretical predictions.

\section{Conclusions}

Based on the results of this study, we demonstrated that the anisotropic elastic constants of a thin unidirec-

Table 1

Lists of the initial guesses and the inverse results of $C_{11}$ and $C_{12}$

\begin{tabular}{llll}
\hline Guess $C_{11}(\mathrm{GPa})$ & Guess $C_{12}(\mathrm{GPa})$ & $C_{11}(\mathrm{GPa})$ & $C_{12}(\mathrm{GPa})$ \\
\hline 222 & 6.0 & & \\
298 & 4.5 & 134.2 & 7.7 \\
254 & 5.5 & & \\
150 & & & \\
200 & 8.0 & 134.8 & 8.2 \\
220 & 7.5 & & \\
135 & 7.2 & & \\
140 & 7.8 & 134.3 & 7.8 \\
148 & 7.2 & & \\
110 & 6.8 & & \\
120 & 8.8 & 134.5 & 8.0 \\
104 & 8.6 & & \\
\hline
\end{tabular}

tional fiber-reinforced composite could be determined using a combination of the ultrasonic bulk wave and Lamb wave. Three out of five elastic constants of the specimen were obtained using the measured pure mode bulk ultrasonic wave velocity. Dispersion of Lamb wave in the thin anisotropic specimen was measured and combined with an inverse algorithm to obtain the rest of the elastic constants. We note that in the present case, only dispersion for a Lamb wave propagating along the fiber direction is intimately related to the elastic constants $C_{11}$ and $C_{12}$. In addition, experimental results demonstrated that the waveform for waves propagating along the fiber direction is very different from that of waves normal to the fiber direction. Finally, we note that with this hybrid method, some of the anisotropic constants can be measured accurately with welldeveloped bulk wave ultrasonics, while the rest of the undetermined elastic constants can be obtained from the inversion of the Lamb wave dispersion.

\section{Acknowledgement}

The authors are grateful for the financial support of this research by the National Science Council of China through Grant NSC87-2732-E-002-002.

\section{References}

[1] R.M. Jones, Mechanics of Composite Materials, Scripta Book Company, Washington, 1975.

[2] S.K. Datta, A.H. Shah, R.L. Bratton, T. Chakraborty, Wave propagation in laminated composite plates, J. Acoust. Soc. Am. 83 (6) (1988) 2020-2026.

[3] W.R. Rose, S.I. Rokhlin, L. Adler, Evaluation of anisotropic properties of graphite composites using Lamb waves, in: D.O. Thompson, D.E. Chimenti (Eds.), Review of Progress in Quantitative Nondestructive Evaluation Vol. 6B, Plenum Press, New York, 1986, p. 1111.

[4] V. Dayal, V.K. Kinra, Leaky Lamb waves in an anisotropic plate. I: an exact solution and experiments, J. Acoust. Soc. Am. 85 (6) (1989) 2268-2276.

[5] A.H. Nayfeh, D.E. Chimenti, Free wave propagation in plates of anisotropic media, J. Appl. Mech. 56 (1989) 881-886.

[6] T.-T. Wu, S.-T. Chiu, On the propagation of horizontally polarized shear waves in a thin composite laminate plate, Ultrasonics 30 (1) (1992) 60-64.

[7] J.F. Chai, T.-T. Wu, Determinations of anisotropic elastic constants using laser generated surface waves, J. Acoust. Soc. Am. 95 (6) (1994) 3232-3241.

[8] M. Veidt, W. Sachse, Ultrasonic evaluation of thin fiber-reinforced laminates, J. Comp. Mater. 28 (4) (1994) 329-341.

[9] R.J. Dewhurst, C. Edwards, A.D.W. Mckie, S.B. Palmer, Estimation of the thickness of thin metal sheet using laser generated ultrasound, Appl. Phys. Lett. 51 (14) (1987) 1066-1068.

[10] D.A. Hutchins, K. Lundgren, A laser study of transient Lamb wave in thin materials, J. Accoust. Soc. Am. 85 (4) (1989) $1441-1448$. 
[11] H. Nakano, S. Nagai, Laser generation of antisymmetric Lamb waves in thin plates, Ultrasonics 29 (1991) 230-234.

[12] S.E. Bobbin, J.W. Wagner, R.C. Cammarata, Interpretation of laser generated low-order Lamb waves for elastic modulus measurements in thin films, Ultrasonics 30 (2) (1992) 87-90.

[13] B.A. Auld, Acoustic Fields and Waves in Solids Vol. 1, Wiley Interscience, New York, 1973.

[14] R.L. Weaver, Y.H. Pao, Axisymmetric elastic waves excited by a point source in plate, J. Appl. Mech. 49 (1982) 821-836.
[15] A.N. Stroh, Steady state problems in anisotropic elasticity, J. Math. Phys. 41 (1962) 77-103.

[16] M.B. Braga, Wave propagation in anisotropic layered composites, Ph.D. dissertation, Stanford University, 1990.

[17] J.A. Nelder, R. Mead, A simplex method for function minimization, Comput. J. 7 (1965) 308-313.

[18] T.-T. Wu, Y.-H. Liu, Inverse analyses of thickness and elastic properties of a bonding layer using laser generated surface waves, Ultrasonics 37 (1999) 23-30. 